\title{
Micro-Sphere Levitation in a Sheath of a Low Pressure Continuous Discharge
}

\author{
C. Arnas, M. Mikikian and F. Doveil
}

Laboratoire de Physique des Interactions Ioniques et Moléculaires, UMR6633 CNRS-Université de Provence, Centre de Saint-Jérôme, case 321, F13397

Marseille, France

\begin{abstract}
Electrode sheath profile measurements are reported, with dust particles in suspension and without With the shape of the unperturbed potential profile, basic characteristics can be predicted as the surface potential, the charge and potential energy of a dust particle in function of its position in the sheath The dust particle screening length is also estimated analyzing binary collisions in a gas phase cloud In the presence of dust particles, an average increase of ion drift velocities is measured showing that micron-sized particles whose charge is due to the electron and ion fluxes produce a selfconsistent redistribution of the plasma particle fluxes
\end{abstract}

\section{Introduction}

Small solid particles immersed in a plasma acquire electric charges [1,2] resulting of electron, ion collection and of secondary electron emission. In a laboratory plasma where the secondary emission due to radiation absorption [3] or to hot particle impacts [4-6] is weak, the dust particle charge is negative. In RF [7] and DC [8] plasmas, when their size is high enough (several microns to tens of microns), dust particles can levitate in the sheath of the lower negative electrode where the gravitation force is balanced by the electric force.

In this paper, we present experiments of dust particle trapping in the sheath of a conducting disc, placed in the center of a DC plasma. Using a differential emissive probe, we first determine the unperturbed electrode sheath profile. The levitation height then provides the sheath electric field and the charge for which a dust particle equilibrium is assured. Knowing the charge, the dust screening length is deduced, by analyzing binary collisions [9] in a dust cloud, in gas phase. On the other hand, using the non intrusive Laser Induced Fluorescence diagnostic, ion drift velocities are measured. From the unperturbed velocities, a sheath profile is deduced as well as the dust particle potential energy, showing the presence of a potential well at a sheath position close to the observed levitation height. In the presence of a dust cloud, an average increase of the ion velocities is observed suggesting that dust grains whose charge is due to the electron and ion fluxes, influence in turn the plasma particle distributions in their neighborhood $[10,11]$.

\section{Experimental set up}

The experiments are performed in a multipolar device, at low argon pressure: $P_{\mathrm{Ar}}=10^{-3} \mathrm{mbar}$ (collisionless sheath). Primary electrons emitted by hot filaments are accelerated toward the grounded wall by a negative voltage. In the plasma center, we have placed a conducting disc (passive electrode), let to the negative floating potential or more negatively biased by an external power supply, to increase the sheath width. The dust particles are hollow glass spheres of mass density $p_{d}=110 \mathrm{~kg} / \mathrm{m}^{3}$ and radius $r_{d}=(32 \pm 2) \mu \mathrm{m}$ or $r_{d}=(22 \pm 2) \mu \mathrm{m}$. Sheath profiles are established perpendicularly to the electrode, using two diagnostics:

i) a differential emissive probe [12], able to measure weak potential variations in pre-sheath and sheath edge regions

ii) the non intrusive Laser Induced Fluorescence (LIF) diagnostic [13] for measurements in the presence of dust particles.

The latter reveals the ion velocity distribution through Doppler effect, applied to the argon ion fluorescence emission, the laser beam propagating perpendicularly to the electrode.

\section{Charging of an isolated dust particle}

The charge $Q_{d}$ of a dust particle is given by $Q_{d}=C \Phi$ where $C \sim 4 \pi \varepsilon_{0} r_{d}$ is the capacitance of a sphere of radius $r_{d}$ and $\Phi$ is the dust particle floating potential, relative to the local potential. The latter parameter is found when for the charging currents on the dust surface, the condition:

$I_{i}(\Phi)+I_{e}(\Phi)+I_{P e}(\Phi)=0$

is achieved, where $I_{i}, I_{e}$ and $I_{p e}$ are respectively the ion, plasma electron and primary electron charging currents. Their expressions are usually given by the Orbit Motion Limited [14] model for which $r_{d}$ must be smaller than the Debye length, in a collisionless plasma-sheath. It is generally assumed that a dust particle in a sheath is charged by monoenergetic ions [15] arriving at the sheath edge with the sound velocity $c_{s}$. The ion flux being conserved, the corresponding current is:

$I_{i}=-\frac{S n_{i} e c_{s}}{4}\left(1-\frac{2 e \Phi}{m_{i} v_{i}^{2}}\right)$

where $S$ is the particle surface, $n_{i}$ and $v_{i}$, the ion density and velocity respectively, depending on the position in the plasma-sheath. In this expression, the ion trapping and reflexion in the orbit trajectories [16] are not taken into account so that Eq. (2) provides the upper limit of the ion current. In the presented experimental conditions, they are two electron populations. The first one is the thermal plasma electron population and the corresponding current is:

$I_{e}=\frac{S}{4} n_{e} e\left(\frac{8 k T_{e}}{\pi m_{e}}\right)^{1 / 2} \exp \left(\frac{e \Phi}{k T_{e}}\right)$

where $T_{e}$ is the electron temperature and $n_{e}$ the electron density depending on the sheath position (Boltzman distribution). The second contribution comes from the primary electrons well defined by an isotropic drifting Maxwellian distribution of drift velocity $v_{D}$. The corresponding current is:

$I_{e p}=\frac{S n_{p e} e}{2 \sqrt{\pi}}\left\{\begin{array}{l}v_{p e}\left[x_{D} x_{p}+1\right] \exp \left(-x_{m}^{2}\right) \\ +v_{D} \sqrt{\pi}\left[x_{m} x_{p}+\frac{3}{2}\right]\left[1+\operatorname{erf}\left(x_{m}\right)\right]\end{array}\right\}(1-\delta)$

where $n_{p e}, T_{p e}$ and $v_{p e}$ are respectively, the primary electron density, temperature and thermal velocity, $x_{D}=v_{D} / v_{p e}, x_{p}=$ 
$\left(v_{D}+v_{m}\right) / v_{p e}, x_{m}=\left(v_{D}-v_{m}\right) / v_{p e}$ and $v_{m}=2 e \phi / m_{e} . \delta$ is the energy dependent secondary emission coefficient [4,6]. The density $n_{p e}$ depends on the sheath position while $x_{p}$ and $x_{m}$ vary with the dust surface potential. Therefore, for each sheath position $[17,18]$, the value of $\phi$ and then $Q_{d}$ can be found. In a first step, if we take as local potential, the plasma potential $V_{p}=-1.7 \mathrm{~V}$, Eq. (1) gives the upper limit of $\phi$. For the plasma parameters measured with a Langmuir probe: $n_{e}$ $=710^{8} \mathrm{~cm}^{-3}, T_{e}=2.5 \mathrm{eV}, n_{p e}=310^{7} \mathrm{~cm}^{-3}, T_{p e}=0.8 \mathrm{eV}$ and the primary electron drift energy $E_{D}=19 \mathrm{eV}$, the surface potential is: $\phi=-17 \mathrm{~V}$. This relatively high negative value, mainly due to the contribution of the primary electrons gives the maximum charge: $\left(Q_{d}\right)_{\mathrm{OML}}=(3.7 \pm 0.3) 10^{5} e^{-}$.

\section{Experimental results and discussion}

\subsection{Dust particle charge}

Figure 1 shows sheath-presheath profiles as a function of $z$, the vertical position above the electrode. Each profile is obtained with a differential emissive probe, for a given electrode bias: the floating potential $-26.5 \mathrm{~V}$ and the biasing voltages $V_{b}=-32,-38,-44$ and $-50 \mathrm{~V}$, assigned by an external power supply. The electrode position is at 0 and the reported experimental data stop at $6.2 \mathrm{~mm}$, the effect of increasing the negative bias being to shift the potential distribution toward the plasma. When the electrode is floating, the plasma potential is reached at about $30 \mathrm{~mm}$. Each measurement set providing data of a collisional presheath (charge exchange between ions and neutrals) and of the beginning of a collisionless sheath is well fitted by the function $V_{s}(z)=\alpha \exp (-\beta z)+\gamma$ where $\alpha$ is equal to the electrode bias and $\gamma$ to the plasma potential. The levitation height in $V_{s}(z)$ gives directly the sheath potential (electric field) for which the electric force $F_{e}=-Q_{d}\left(\partial V_{s} / \partial z\right)$ balances the gravitation force $F_{g}=m_{\mathrm{d}}$ g.

Injecting a single dust particle in the plasma, we observe that when the electrode bias is negatively increased, the levitation height $h$ (Table I) increases too, from $1.9 \mathrm{~mm}$ for the floating electrode case to $3.34 \mathrm{~mm}$ when $V_{b}=-50 \mathrm{~V}$. Each position provides almost the same equilibrium sheath potential (relatively to the ground) and electric field. The mean local electric field is $E_{e q}=\left(\begin{array}{ll}2.0 \pm 0.3\end{array}\right) 10^{3} \mathrm{~V} / \mathrm{m}$. Therefore, the equality $F_{e}=F_{q}$ gives the charge $Q_{d}=(4.4 \pm$ $0.7) 10^{5} e^{-}$of the same order as $\left(Q_{d}\right)_{\mathrm{OML}}$.

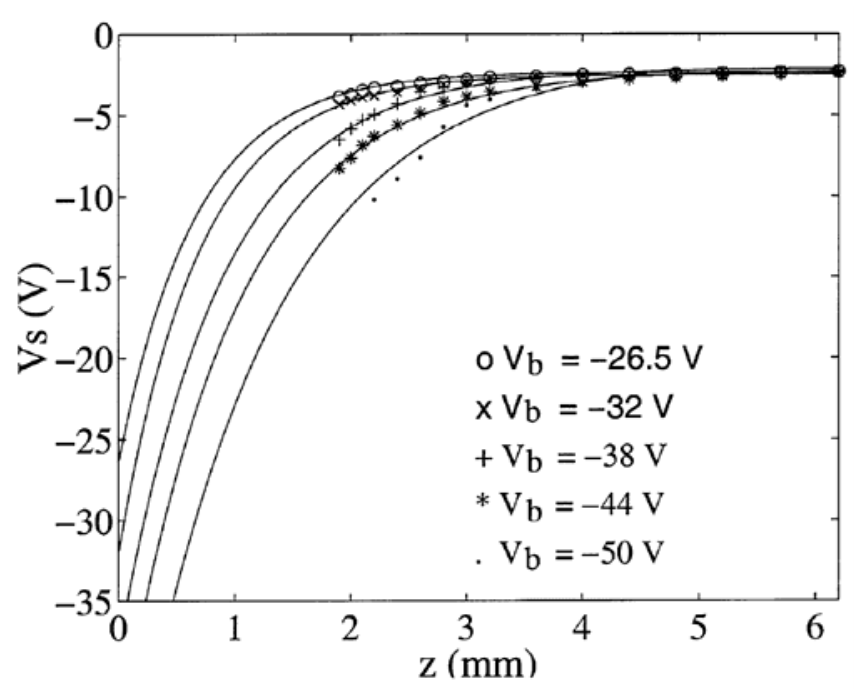

Fig. 1 Electrode potential profiles for electrode biases of -26.5, -32, -38, $-44,-50 \mathrm{~V}$

\begin{tabular}{|cccccc||}
\hline $\mathbf{V}_{\mathbf{b}}($ Volts) & $\mathbf{- 2 6 . 5}$ & $\mathbf{- 3 2}$ & $\mathbf{- 3 8}$ & $\mathbf{- 4 4}$ & $\mathbf{- 5 0}$ \\
\hline \hline $\mathbf{h}(\mathbf{m m})$ & 1.9 & 2.3 & 2.6 & 2.9 & 3.3 \\
$\mathbf{V}_{\text {eq }}($ Volts) & -3.9 & -3.6 & -3.9 & -4.2 & -4.4 \\
$\mathbf{E}_{\text {eq }}(\mathbf{k V} / \mathbf{m})$ & 2.1 & 1.6 & 2.1 & 2.2 & 2.4 \\
$\mathbf{Q}_{\mathbf{d}}\left(\mathbf{1 0}^{\mathbf{5}} \mathbf{e}\right)$ & 4.3 & 5.6 & 4.3 & 4.2 & 3.8 \\
\hline
\end{tabular}

Table I. For a given electrode bias $V_{b}$, h is the levitation height, $V_{e q}$ and $E_{e q}$ are the corresponding sheath potential and electric field and $Q_{d}$, the deduced dust particle

\subsection{Dust particle screening length}

An analysis of a binary collision is presented, assuming the presence of a screened Coulomb potential around each particle. At $10^{-3} \mathrm{mbar}$ and low discharge current, dust particles trapped in the electrode sheath form a single layer cloud, parallel to the electrode. Their trajectories follow straight lines with direction changes in the horizontal plane, resulting from their collisions (gas phase). Figure 2(a) shows successive binary collisions of four particles. Particle 1, coming from the bottom is deviated toward the left side after its collision with particle 2, initially motionless. The latter is accelerated and after diffusing on the third particle, 2 is deviated to the left side. Particle 4 is then deviated to the right side after its interaction with 3 . Figure 2(b) is a magnification of the collision between the incident particle 1 and the target particle 2 . The trajectories are materialized by dotted lines. It is assumed here, that there is no loss charge during the collision and that polarization forces are negligible, so that the particle interaction is only due to a central force. In this case, the scattering of a charged particle on another initially motionless, provides the following equation:

$\tan \chi_{0}=\frac{\sin 2 \psi_{0}}{\frac{m_{1}}{m_{2}}-\cos 2 \psi_{0}}$

where $\chi_{0}$ is the deviation angle of the particle 1 and $\psi_{0}$ is the particle 2 trajectory angle, after the collision. These angle measurements provide the mass ratio $R=m_{1} / m_{2}=0.93$. So, in the limit of our dust size dispersion, for the forthcoming analysis, the incident particle radius $r_{1}$ is taken equal to 31 $\mu \mathrm{m}$ while $r_{2}$ (target radius) is deduced from $R$. With the corresponding masses, projecting the measured constant velocities before and after the collision (far enough from the collision) on two perpendicular axes, we check that there is conservation of the momentum and the kinetic energy, confirming the assumption of elastic collision. During the collision, the interaction potential between the particles must be taken into account. The conservation of the angular momentum and of the total energy yields in $r_{m}$ (minimum approach distance), the trajectory equation:

$1-\frac{p^{2}}{r_{m}{ }^{2}}-2 \frac{\varphi\left(r_{m}\right)}{\mu v_{1}{ }^{2}}=0$

where $p$ is the particle 1 impact parameter, $v_{1}$ its velocity before the collision, $\mu$ the reduced mass and $\varphi$ the interaction potential between both particles. Eq. (6) must be fulfilled whatever the shape of $\varphi$. Assuming a screened Coulomb interaction potential, $\varphi(r)$ is given by: 
$\varphi(r)=\frac{Q_{1} Q_{2}}{4 \pi \varepsilon_{0} r} \exp \left(-\frac{r}{\lambda_{S}}\right)$

where $Q_{1,2}$ is the charge of the incident (target respectively) particle and $\lambda_{s}$, the screening length. The measured values are: $p=(300 \pm 20) \mu \mathrm{m}, r_{m}=(670 \pm 40) \mu \mathrm{m}, v_{1}=(50 \pm 10)$ $\mathrm{mm} / \mathrm{s}$. Eq. (1) giving a floating potential of $-14 \mathrm{~V}$, the charges are $Q_{1}=(3.0 \pm 0.3) 10^{5} e^{-}$and $Q_{2}=(3.3 \pm 0.3) 10^{5} e^{-}$ where the errors bar come from the size dispersion and from an assumed error of $1 \mathrm{~V}$ for the floating potential. The screening length is then $\lambda_{\mathrm{s}}=(0.46 \pm 0.12) \mathrm{mm}$. The electron and ion densities can be calculated for each sheath position so that, at the levitation height, the electron Debye length is $\lambda_{D e} \sim 0.6 \mathrm{~mm}$ and the ion Debye length, $\lambda_{\mathrm{Di}} \sim 0.09 \mathrm{~mm}, \lambda_{\mathrm{s}}$ being of the order of $\lambda_{\text {De }}$ [9]. As we will see in the following section, at the levitation height, the ions are supersonic: being unable to screen the dust particle charge [19,17], $\lambda_{\mathrm{Ds}} \sim$ $\lambda_{\text {De }}$ shows the lack of electrons in the dust particle surrounding.
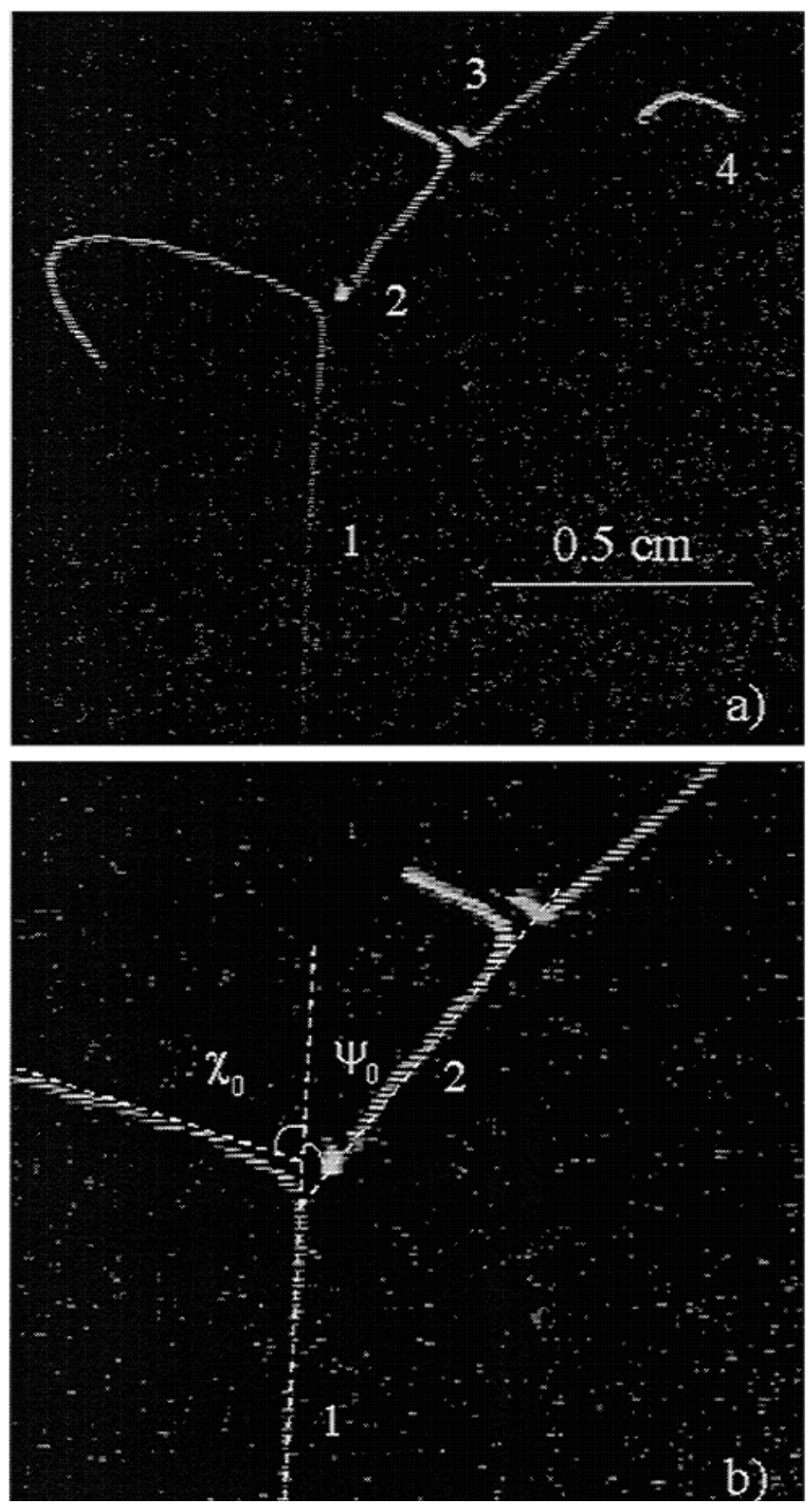

Fig 2. (a) Successive binary collisions between four dust particles (b) Magnification of the left bottom side collision.

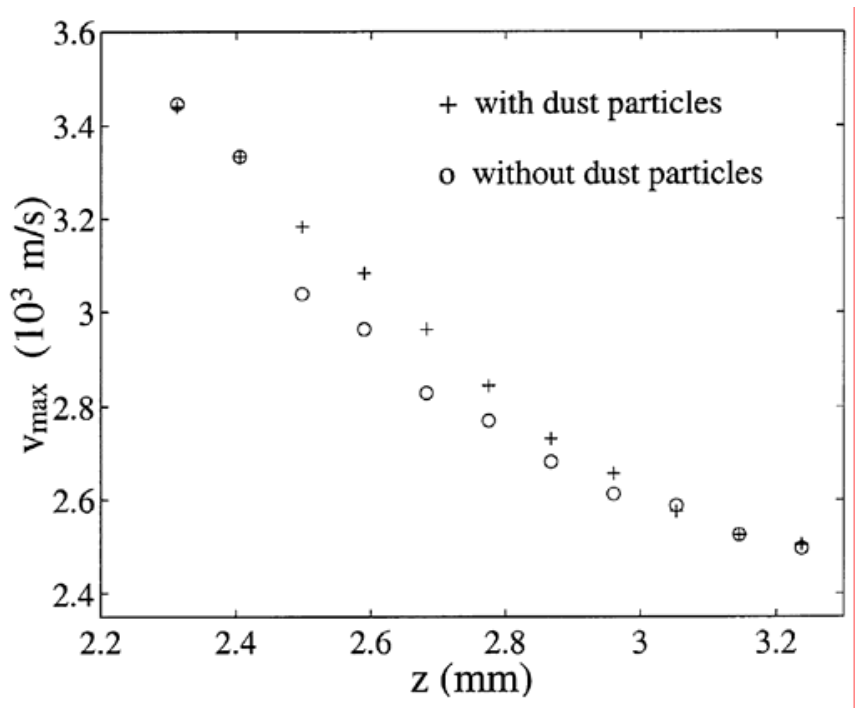

Fig. 3. Ion drift velocity $v_{\max }$ corresponding to the maximum of the ion velocity distribution, without and with dust particles, versus the position in the sheath layer where dust particle effects are observed.

\subsection{Ion velocity distribution and consequences}

At $10^{-3} \mathrm{mbar}$, when the discharge current is high enough, the particles oscillate vertically during their horizontal motion. We have chosen this situation for two reasons:

i) with a relative high discharge current, the fluorescence signal is detected easily above the noise level

ii) the effect of the dust particles on the ions is increased when they oscillate in the ion flow direction.

In these conditions, from six to eight dust particles $\left(r_{d}=\right.$ $22 \mu \mathrm{m})$ are in the laser beam at any time. As the collection system of the fluorescence signal is moved vertically toward the electrode, the ion velocity distribution $f\left(v_{i}\right)$ is shifted toward increasing ion velocities [13]. Figure 3 gives $v_{\max }$, the ion drift velocity corresponding to the maximum of $f\left(v_{i}\right)$, versus the position, from $2.3 \mathrm{~mm}$ to $3.2 \mathrm{~mm}$, the sheath layer in which the cloud effects are observed. The open circles represent the measurements without dust particles whereas the crosses are obtained in their presence. In spite of our limited spatial resolution (small volume occupied by the dust grains with respect to the fluorescence emissive volume), these measurements show that the drift velocities are higher when there are dust particles (qualitative ion acceleration). With $T_{e}=2.2 \mathrm{eV}$, the ion acoustic velocity is $c_{s} \sim 2.310^{3} \mathrm{~m} / \mathrm{s}$. So, the particle trapping occurs in a sheath layer where the ions are supersonic.

The energy of an ion of velocity $v_{\max }$, in the sheath potential $V_{s}$ is $E=1 / 2 m_{i} v_{i}^{2}+e V_{s}$. The energy conservation yields: $E=e V_{p}$ where $V_{p}$ is the plasma potential. The deduced $V_{s}(z)$ is shown in the left scale of Fig. 4(a). The open circles are the data without particle, fitted (solid line) by $V_{s}(z)=\alpha \exp (-\beta z)+\gamma$ as in Section 4.1. Here, $\gamma=0$ since the data are referenced with respect to the value $V_{s}=$ $2.7 \mathrm{~V}$, obtained at $\mathrm{z}=3.2 \mathrm{~mm}$. Eq. (1) provides the dust surface potential $\varphi(z)$, relative to the potential $V_{s}(z)$ (dot curve and right scale in Fig. 4(a)). Figure 4(b) gives the potential energy profile, due to the gravitation and the electrostatic forces: $U(z)=-m_{d} g z+Q(z) V_{s}(z)$. 

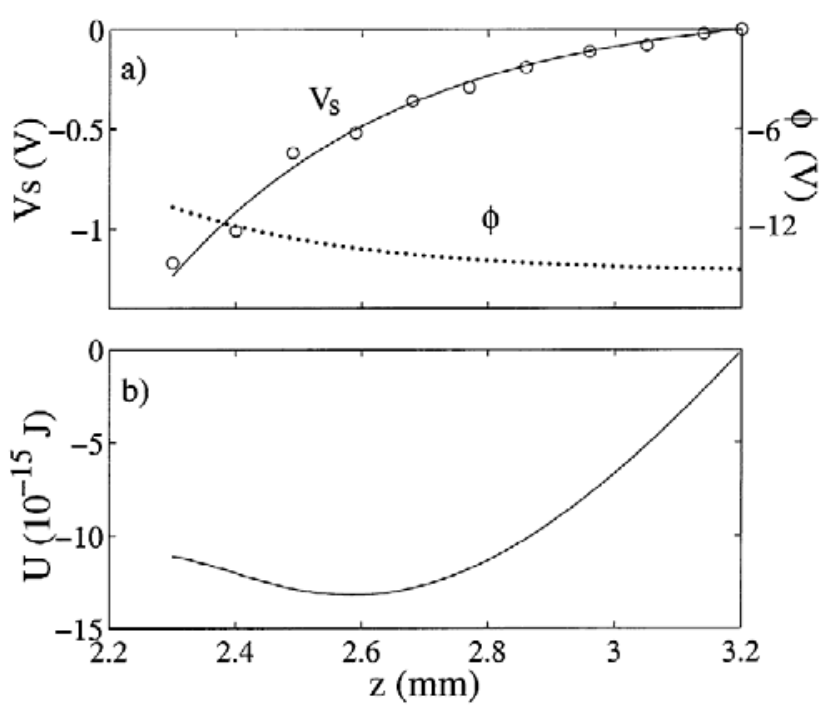

Fig. 4 (a) Sheath layer profile $V_{s}$ (left scale) and dust particle surface potential $\varphi$ (right scale) versus the position. (b) Dust particle potential energy showing a potential well, versus the position.

A potential well appears in $z_{0} \sim 2.6 \mathrm{~mm}$, close enough to the observed levitation height: $h_{0}=(2.7 \pm 0.1) \mathrm{mm}$ and justifying dust oscillations in this region. In $z_{0}$, the dust surface potential is $\varphi=-13 \mathrm{~V}$ (Fig. 4(a)), providing the charge: $Q_{d}=210^{5} e^{-}$. At the same location, the gravitation and electric force balance gives $Q_{d}=1.810^{5} e^{-}$, of the same order. Nevertheless, for a more accurate analysis, we should use the real potential profile due to the plasma particle redistribution in the presence of dust particles.

\subsection{Potential profile in the presence of a dust cloud}

Figure 5 gives the potential profiles with respect to the grounded wall, in the presence of dust particles (crosses) and without (open circles). The negative potential values are decreased, in the presence of dust particles. However, as said previously, because of a limited spatial resolution, the LIF diagnostic only reveals a qualitative decrease of the negative sheath potential. In particular, we cannot observe any potential jump due to the dust surface potential (-13 V) as well as potential change due to the ion focusing downstream to the dust particles [10]. This difficulty is increased by the fact that having a large velocity compared to the ion thermal velocity, the ions are unable to screen the dust grains. Our results show at least that if micron-sized particles are charged by electron and ion fluxes, they influence in turn the plasma particle distributions. This selfconsistent behavior is not described by the widely used charging model given in Section 3. However, this latter is assumed to provide the dust parameters in a reasonable approximation.

\section{Conclusion}

In continuous discharge conditions, sheath profile measurements have been presented from which the charge of an isolated dust grain can be deduced:

i) the levitation height provides the potential (electric field) where the gravitation force is balanced by the electric force

ii) the dust potential energy calculation as a function of the sheath position allows us to find a trapping potential well, the charge being found at the well position.

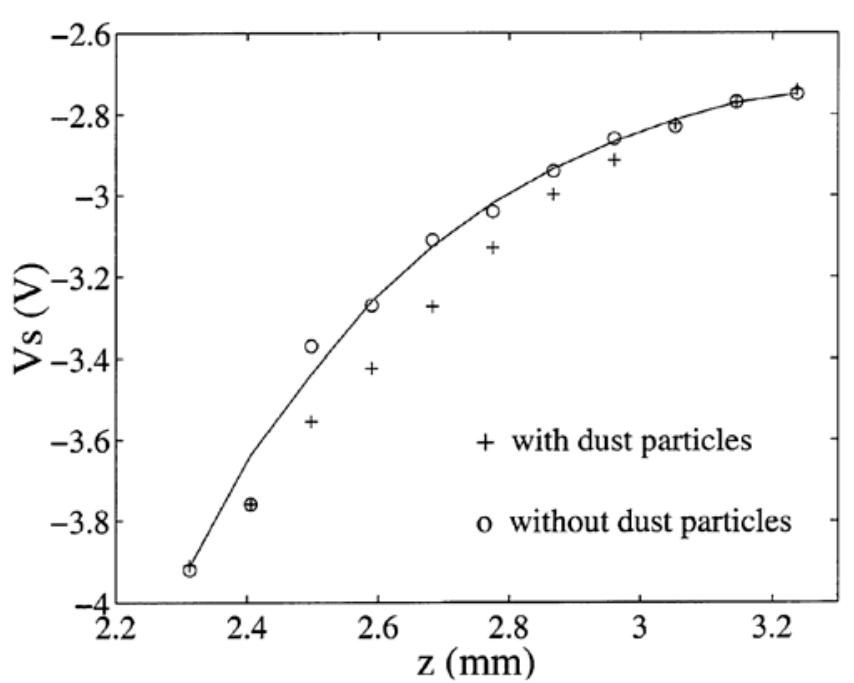

Fig. 5 Sheath layer profiles without and with dust particles

Knowing the charge, the particle screening length is deduced by analyzing binary collisions in the situation of a dust cloud in gas phase. In the other hand, we have measured an increase of the ion drift velocities, in the vicinity of the levitation height. The deduced sheath profile modification shows that micron-sized particles, charged by electron and ion fluxes, produce a self-consistent redistribution of the surrounding plasma particle fluxes.

\section{Acknowledgements}

The authors thank V Tsytovich, S Vladimirov and A Bouchoule for helpful discussions This work was supported by a grant of the Direction des Recherches et Etudes Techniques

\section{References}

1 Whipple, E.C., Rep. Prog. Phys. 44, 1197 (1981)

2 Goertz, C.K., Rev. Prog. Phys. 27, 271 (1989)

3 Havnes, O., Adv. Space Res. 4, 75 (1984)

4 Sternglass, E.J., Sci. Pap. 1772, Westinghouse Res. Lab., Pittsburg, Pa. (1954)

5 Meyer-Vernet, N., Astron. Astrophys. 105, 98 (1982)

6 Walch, B., Horanyi, M. and Robertson, S. , Phys. Rev. Lett. 75, 838 (1995)

7 Selwyn, G.S., Singh, J. and Bennet, R.S., J. Vac. Sci. Technol. A 7, 2758 (1988)

8 Arnas, C. , Mikikian, M. and Doveil, F., Phys. Rev. E 60, 7420 (1999)

9 Konopka, U., Ratke, L. and Thomas, H.M., Phys. Rev. Lett. 79, 1269 (1997); Konopka, U., Morfill, G.E. and Ratke, L., Phys. Rev. Lett. 84, 891 (2000)

10 Melandso, F. and Goree, J., Phys. Rev. E 52, 5312 (1995)

11 Benkadda, S., Tsytovich, V.N. and Vladimirov, S.V., Phys. Rev. E 60, 4708 (1999)

12 Yao, W. En, Intrator, T. and Hershkowitz, N., Rev. Sci. Instrum. 56, 519 (1985)

13 Goeckner, M.J., Goree, J. and Scheridan, T.E., Phys. Fluids B4, 1663 (1992)

14 Laframboise, J.G. and Parker, L.W., Phys. Fluids 16, 629 (1973)

15 Barnes, M.S., Keller, J.H., Forest, J.C., O'Neil, J.A. and Coultas, D.K., Phys. Rev. Lett. 68, 313 (1992)

16 Bernstein, I.B. and Rabinowitz, I.N., Phys. Fluids 2, 112 (1959)

17 Nitter, T., J. Vac. Sci. Technol. 5, 93 (1996)

18 Nunomura, S., Ohno, N. and Takamura, S., Phys. Plasmas 5, 3517 (1998)

19 Hamaguchi, S. and Farouki, R.T., Phys. Rev. E 49, 4430 (1994) 